\title{
Commercialisation du caoutchouc naturel des plantations villageoises : un facteur essentiel du développement de l'hévéaculture au Cameroun
}

\section{Bénédicte Chambon \\ Jean-Marie Eschbach \\ Cirad}

Département «Performances des systèmes de production et de transformation tropicaux " UR « Performance des systèmes de cultures des plantes pérennes "

TA B-34/02, Avenue Agropolis 34398 Montpellier CEDEX 5

<benedicte.chambon@cirad.fr> <jean-marie.eschbach@cirad.fr>

\begin{abstract}
Résumé
Dans cette étude, nous nous interrogeons sur la manière dont les conditions de commercialisation du caoutchouc naturel influencent le développement du secteur hévéicole villageois au Cameroun. Pour cela, nous analysons les résultats de deux enquêtes réalisées en 2002 et 2004 auprès de petits planteurs d'hévéa dans la province SudOuest. Les résultats montrent que les conditions de commercialisation observées conduisent à des pratiques qui ont des conséquences financières défavorables pour les planteurs, notamment en terme de trésorerie. Même si, durant la période étudiée, les problèmes ont été accentués par le bas niveau de prix jusqu'en 2002, garantir de bonnes conditions de commercialisation apparaît comme une étape essentielle et un préalable obligé du développement des plantations villageoises d'hévéa.
\end{abstract}

Mots clés : Cameroun ; caoutchouc naturel ; commercialisation ; exploitation agricole familiale ; Hevea brasiliensis.

Thèmes : économie et développement rural ; productions végétales ; systèmes agraires ; transformation, commercialisation.

\section{Abstract \\ Marketing of smallholder natural rubber: An essential factor in the development of Cameroonian rubber-growing}

In this study, we investigated how natural rubber marketing conditions affect the development of rubber smallholdings in Cameroon. To that end, we analysed the results of two surveys, conducted in 2002 and 2004, among rubber smallholders in Southwest province. The results showed that the observed marketing conditions led to practices with unfavourable financial consequences for farmers, notably in terms of cash flow. Although problems were exacerbated during the study period by low price levels up to 2002, guaranteeing good marketing conditions appears to be a prerequisite for the development of rubber smallholdings.

Key-words: Cameroon; family farms; Hevea brasiliensis; marketing; natural rubber.

Subjects: economy and rural development; farming systems; processing, marketing; vegetal productions.

n 2006, le Cameroun était le $11^{\mathrm{e}}$ producteur mondial de caoutchouc naturel avec 62000 tonnes, soit $0.67 \%$ de la production mondiale, principalement concentrée en Asie (IRSG, 2007). Alors que $80 \%$ du caoutchouc mondial provient de plantations villageoises, le secteur hévéicole camerounais a toujours été largement dominé par les plantations industrielles. La production des planta- tions villageoises reste limitée pour deux raisons : leur productivité $(900 \mathrm{~kg} / \mathrm{hec}-$ tare) bien inférieure à celle des plantations industrielles (1 $500 \mathrm{~kg} /$ hectare) et la faible superficie plantée (4 000 hectares, soit près de $10 \%$ de la surface totale hévéicole au Cameroun), ce malgré un important potentiel (Gobina et al., 2002). L'hévéa constitue une opportunité intéressante pour améliorer les conditions 
de vie des exploitations familiales. Il représente une solution alternative aux autres cultures pérennes (cacaoyer et palmier à huile) et aux cultures vivrières destinées en partie à la vente (manioc, plantain...), avec lesquelles elle est aussi souvent complémentaire.

Les conditions socio-économiques (accès au crédit, matériel végétal, encadrement technique, commercialisation...) dans lesquelles les exploitations familiales évoluent sont importantes pour le développement des plantations villageoises d'hévéa. On s'intéresse ici à l'une de ces conditions, la commercialisation du caoutchouc naturel, et on s'interroge en particulier sur la manière dont ces conditions influencent le développement du secteur villageois. Nous faisons l'hypothèse que les conditions de commercialisation sont un facteur de blocage du développement des plantations villageoises et, plus précisément, que des conditions de commercialisation défavorables aux petits planteurs ont des conséquences sur leurs pratiques hévéicoles et sur la production.

\section{Quelques repères}

\section{sur l'hévéaculture}

\section{et le secteur hévéicole}

\section{camerounais}

Le caoutchouc naturel est prélevé sur l'hévéa par des saignées répétées au cours de l'année. La saignée est seulement interrompue pendant la période de refoliation des arbres (un mois). L'hévéa génère donc un revenu régulier tout au long de l'année. La saignée nécessite beaucoup de main-d'œuvre, puisqu'en milieu paysan, elle a généralement lieu tous les deux ou trois jours (plus rarement tous les quatre jours). C'est une technique difficile, qui demande un savoir-faire.

Au Cameroun, le caoutchouc des plantations villageoises est commercialisé sous forme de coagulums (coagulation du latex dans le récipient de récolte), dont la teneur en caoutchouc sec (ou DRC, Dry rubber content) est de l'ordre de $50 \%$ à la récolte - elle augmente avec le stockage. Le prix du caoutchouc est fixé par kilogramme de caoutchouc sec; le DRC a donc une importance pour la détermination du prix payé aux planteurs. Les coagulums doivent subir un traitement post-récolte pour être valori- sés. Or, au Cameroun, ce traitement n'est réalisé que dans les usines des groupes agro-industriels.

Trois agro-industries possèdent des plantations d'hévéas: une société parapublique, la Cameroon Development Corporation (CDC) dans la province Sud-Ouest (20 000 hectares) et deux sociétés privées, Safacam dans le Littoral (3 500 hectares) et Hevecam (Hévéa du Cameroun) dans le Sud (17 000 hectares). L'essentiel des plantations villageoises a été créé entre 1978 et 1992, soit dans le cadre du projet Fonader - projet de développement des plantations villageoises (environ 1300 hectares dans le SudOuest et le Sud) -, soit à l'initiative des paysans (planteurs non encadrés, environ 2000 hectares, principalement dans le Sud-Ouest). Il existe aussi, dans les provinces Sud et Est, des plantations datant de l'époque coloniale. La CDC et Hevecam ont été les opérateurs techniques du projet Fonader respectivement pour le Sud-Ouest et le Sud. Elles étaient notamment chargées de la collecte, de l'usinage des coagulums et de la rémunération des planteurs.

\section{Méthodologie}

L'étude menée en collaboration avec l'Irad (Institut de recherche agricole pour le développement) a été réalisée dans la province Sud-Ouest, plus précisément dans le bassin d'approvisionnement de la CDC (figure 1). Cette zone concentre $84 \%$ des surfaces de plantations villageoises d'hévéa exploitées au Cameroun. Nous nous appuyons sur deux enquêtes dont les données ont été interprétées à l'aide du logiciel SPHINX-Plus.

La première enquête a été réalisée en 2002 chez 26 planteurs et la deuxième en 2004 chez 56 planteurs (12\% des producteurs recensés dans la zone). Des planteurs encadrés par le projet Fonader et non encadrés ont été sélectionnés de façon aléatoire dans la liste fournie par la CDC (tableau 1). Les enquêtes se déroulaient en anglais, en présence le plus souvent d'un encadreur qui servait si nécessaire de traducteur dans le dialecte local. Le questionnaire était adressé au chef d'exploitation, mais il est arrivé que ce soit un de ses enfants ou le responsable de la saignée qui réponde aux questions.

Pour l'enquête de 2004, une période de trois ans (2000-2003) est considérée. Du fait des nombreuses questions ouvertes, les informations obtenues sont assez hétérogènes. Par ailleurs, des questions sur le stockage de la production n'ont été ajoutées qu'après le début de la collecte des données ; seulement 28 planteurs (sur les 56) y ont donc répondu. Sur la base des réponses données par les

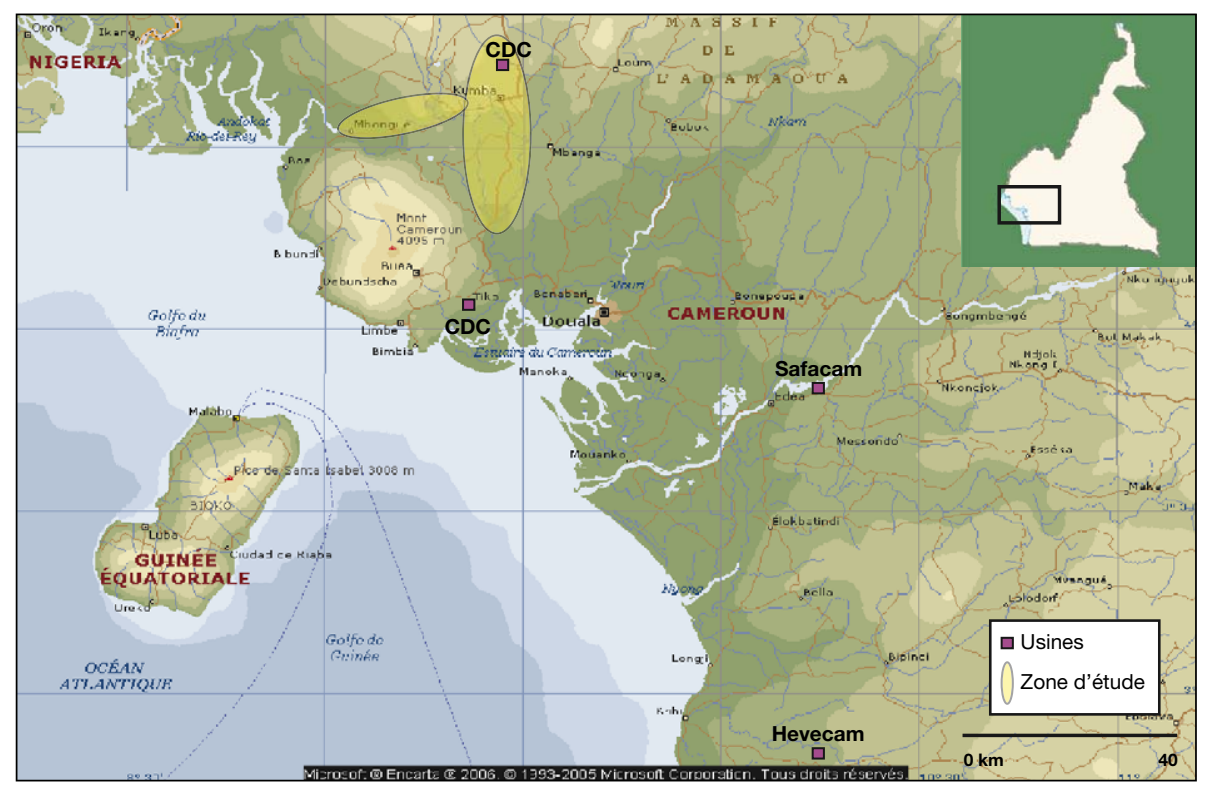

Figure 1. Zone d'étude.

Figure 1. Study zone

CDC: Cameroon Development Corporation. 
Tableau 1. Composition des échantillons.

Table 1. Sampling.

\begin{tabular}{lcccc}
\hline & Année & $\begin{array}{c}\text { Planteurs } \\
\text { encadrés }\end{array}$ & $\begin{array}{c}\text { Planteurs } \\
\text { non encadrés }\end{array}$ & Total \\
\hline Enquête 1 & 2002 & 16 & 10 & 26 \\
Enquête 2 & 2004 & 32 & 24 & 56 \\
\hline
\end{tabular}

planteurs, les questions ouvertes ont été transformées en questions fermées, pour pouvoir faire l'objet d'une analyse statistique. Toutefois, certaines données n'ont pas été traitées statistiquement: elles donnent des informations sur un phénomène ou une pratique sans quantifier son importance (par exemple, on ne connaît pas le pourcentage de planteurs concernés). Cette limite est liée au fait que l'objectif de cette enquête était d'étudier les raisons de la faible production des villageois ; il ne s'agissait pas d'une enquête spécifique sur la commercialisation.

Une autre limite à souligner est la faible taille des échantillons, en particulier pour l'enquête de 2002. Les 26 planteurs correspondent à une partie de l'échantillon total d'une enquête plus vaste (sur deux zones d'étude). Ce nombre est faible pour une analyse statistique des données, mais suffisant pour donner une idée de la problématique.

L'analyse des fiches de la CDC recensant les productions mensuelles de chaque petit planteur a fourni des informations sur la livraison.

\section{Résultats}

\section{Une production irrégulière du caoutchouc naturel}

Dans la plupart des exploitations (73\%), la saignée est assurée par de la maind'œuvre extérieure à la famille, bien que $65 \%$ des paysans maîtrisent la technique. Les saigneurs sont généralement rémunérés sur la base du nombre de kilogrammes de caoutchouc humide produits $(0,08$ euro $/ \mathrm{kg}$ soit $14 \%$ du prix du caoutchouc sec payé au planteur en 2002) ou, plus rarement à la journée de travail (2,30 euros/jour). Le métayage est peu répandu (20\% des saigneurs) ; le métayer reçoit le revenu de $50 \%$ de la production, après déduction du coût des intrants et du transport.
Beaucoup de chefs d'exploitation ont des difficultés pour rémunérer leurs saigneurs. Parmi eux, $26 \%$ seulement assurent une rémunération mensuelle. Quelques planteurs (21\%) donnent quand même chaque mois une avance sur salaire comprise entre 15 et 46 euros en fonction du niveau de production et des besoins des saigneurs (15 à $50 \%$ du salaire mensuel). Malgré le fait que l'hévéa génère théoriquement un revenu régulier, près de la moitié des planteurs ont recours au crédit pour payer les saigneurs.

Sur la période étudiée (2000-2003), 60 \% des paysans ont momentanément interrompu la saignée sur toutes leurs plantations en dehors de la période de refoliation. La saignée a parfois été arrêtée plusieurs mois (11\%), voire plusieurs années (16\%). Mais, le plus souvent, elle a été irrégulière avec de courtes périodes d'interruption (33\%). Proportionnellement, les arrêts de saignée se rencontrent plus dans les exploitations où elle est assurée par la main-d'œuvre familiale $(78 \%)$, que dans celles ayant recours à un saigneur (52\%).

Près de la moitié des planteurs justifient les arrêts de saignée par un problème de main-d'œuvre. Seulement $40 \%$ des paysans enquêtés réussissent à garder les mêmes saigneurs. Dans certains villages proches des plantations de la CDC, beaucoup de paysans (plus de 50 \%) subissent la concurrence de l'agro-industrie, les saigneurs préférant travailler pour cette structure qui paie régulièrement ses employés.

Enfin, pour assurer une rémunération régulière des saigneurs et tenter ainsi de les fidéliser, certains chefs d'exploitation réduisent le nombre d'employés. Une partie de la plantation est ainsi laissée inexploitée.

\section{Une commercialisation difficile des coagulums}

La plupart des paysans (60\%) assurent eux-mêmes le transport de leur caoutchouc vers les usines de transformation, puisque la CDC a quasiment cessé la collecte dans les villages depuis la fin des années 1990 (activité peu rentable). Il n'existe pas réellement de réseau de collecte de la production dans les villages : moins de $4 \%$ des planteurs vendent parfois leur caoutchouc à un commerçant privé. En revanche, ils sont un peu plus de $20 \%$ à le vendre ponctuellement à des hévéaculteurs du village. Dans 90 \% des cas, cela correspond à un besoin urgent de liquidités. Cette solution présente un double avantage: un paiement au moment de la vente et pas de transport dont il faille avancer les fonds puisque celui-ci est assuré par l'acheteur. Cependant, le prix reçu est bas : il est 22 à $50 \%$ inférieur au prix payé par la CDC pour une production livrée à l'usine.

Le taux de remplissage des véhicules qui livrent la production individuelle est en moyenne de $40 \%$. Malgré cela, un peu moins de la moitié seulement des planteurs groupent toujours leur production avec d'autres paysans et $17 \%$ le font de manière occasionnelle. Ces regroupements se font entre voisins et amis, et non dans le cadre d'organisations paysannes formalisées.

Dans le cadre du projet Fonader, la vente du caoutchouc villageois était prévue à la fin de chaque mois. Or, la fréquence observée de livraison de la production aux usines de la CDC est de 6-7 livraisons/an. Quasiment $80 \%$ des planteurs interrogés stockent la production pendant deux, voire trois mois pour deux raisons : une production trop faible, donc un coût unitaire du transport trop élevé, et une trésorerie non disponible pour financer le transport.

Les planteurs sont rémunérés sur la base d'un DRC fixe de $58 \%$, quelle que soit la durée du stockage.

Les retards de paiement de la production par la CDC sont importants, de trois mois en moyenne, parfois quatre. Ils sont une contrainte pour les planteurs : $66 \%$ expliquent ainsi leur faible niveau de production.

Les principaux résultats à retenir de cette étude conduite au début des années 2000 sont :

- le recours à de la main-d'œuvre rémunérée pour la saignée, ce qui entraîne des besoins de financement;

- les interruptions de saignée liées à un problème de trésorerie pour rémunérer la main-d'œuvre ;

- des conditions de commercialisation défavorables aux planteurs: difficultés pour assurer le transport, DRC bas, longs délais de paiement de la production. 


\section{Discussion}

Les résultats montrent que les conditions de commercialisation expliquent certaines pratiques des paysans ayant des conséquences sur le niveau de production des plantations et le revenu des exploitations. Trois caractéristiques interviennent dans les conditions de commercialisation.

D'abord la situation de monopole d'achat par la CDC lui permet de fixer les conditions de la transaction, notamment les délais de paiement et le DRC. Les longs délais de paiement sont une contrainte forte pour les planteurs en termes de trésorerie : problème de rémunération des saigneurs, donc de disponibilité de la maind'œuvre, avec des conséquences sur la production ; problème aussi pour financer le transport du caoutchouc, donc irrégularité des livraisons. Ces délais ne se rencontrent pas à Hevecam ni dans d'autres pays africains (Gabon, Ghana) où il existe pourtant aussi une situation de monopole. De plus, le DRC fixe de $58 \%$ désavantage les planteurs ; il correspond au DRC de coagulums 3 jours après la saignée, or il passe à $70 \%$ environ après 15 jours de stockage au champ (Place, 2003). Ce problème de DRC se retrouve dans la zone d' Hevecam.

Par ailleurs, la non-organisation des producteurs a deux conséquences. D'abord, au niveau des villages, l'absence d'organisation formalisée prive les planteurs d'une structure qui pourrait faciliter certaines activités hévéicoles (comme le transport de la production). Ensuite, ils n'ont aucun pouvoir de négociation avec la CDC, ce qui paraît d'autant plus défavorable que les planteurs sont face à un acheteur unique.

Enfin, l'arrêt de la collecte du caoutchouc villageois par la CDC (contrairement à Hevecam qui continue à l'assurer dans sa zone d'intervention) et la quasiabsence de collecteurs privés obligent les planteurs à organiser et à avancer les fonds du transport, ce qui est souvent une contrainte. Dans de nombreux pays, les paysans peuvent vendre leur production à des intermédiaires présents dans les villages. Ce système domine dans beaucoup de pays asiatiques : Indonésie (Sardjono, 2005), Malaisie (Hashim, 2005), Thaillande (Buakaew, 2005). On le rencontre aussi en Côte d'Ivoire depuis la libéralisation de la filière hévéicole en 1996 (Kpolo, 2001). L'existence d'un réseau d'intermédiaires présente plusieurs avantages pour les paysans (Gouyon et Supriono, 1993). Cependant, la longueur de la chaîne peut augmenter les coûts de commercialisation (Uhendi Haris et Teguh Wahyudi, 2005). $\mathrm{Au}$ Cameroun, le désengagement de la CDC de l'activité de collecte du caoutchouc villageois n'a pas conduit au développement d'un réseau de commerçants privés comme il en existe, par exemple, pour le cacao. Plusieurs hypothèses peuvent être formulées sur les raisons de cette situation: marché insuffisant, rôle de la CDC (donc de l'État), relations entre les planteurs et l'agro-industrie. Les résultats de nos enquêtes ne permettent pas de privilégier l'une ou l'autre de ces hypothèses ; une étude complémentaire serait donc nécessaire.

Les pratiques liées aux conditions de commercialisation ont des conséquences financières pour les exploitations familiales.

D'abord les planteurs ont dû renoncer à un revenu à court terme, alors qu'ils disposent du capital productif potentiel ; la perte de revenu, en moyenne de 500 euros/hectares/an, peut aller jusqu'à 750 euros/hectares/an (Plaza, 2003). À long terme, les arrêts de saignée observés ne pénalisent pas les paysans, puisqu'un hévéa non exploité continue à augmenter son capital théorique de production (l'arrêt de saignée permet l'accumulation de réserves hydrocarbonées favorables à la croissance de l'arbre et à la production future).

De plus, le stockage du caoutchouc prive les planteurs du revenu mensuel (intérêt reconnu de l'hévéa) et donc de la trésorerie nécessaire pour subvenir aux besoins de la famille et financer les activités agricoles courantes. Dans les exploitations familiales hévéicoles du Sud-Ouest du Cameroun, les cultures pérennes contribuent fortement au revenu de l'exploitation - 58 à $82 \%$ selon le type d'exploitation - et l'hévéa y occupe une place importante - 31 à $62 \%$ - (Chambon et al., 2007). On comprend donc les problèmes de trésorerie que les délais de paiement du caoutchouc peuvent créer. En revanche, contrairement à d'autres cultures, le stockage du caoutchouc comme le font les planteurs camerounais n'entraîne pas de perte de qualité du produit. Le stockage au-delà d'un mois ne pénalise pas les paysans au niveau du montant du revenu généré, puisque le DRC plafonne à $75 \%$ après trois semaines Enfin, la vente du caoutchouc à des "planteurs intermédiaires " est très désavantageuse pour le producteur. La marge de l'acheteur est importante, puisque le coût du transport est estimé à $5 \%$ du prix du caoutchouc sec payé par la CDC à partir de $30 \mathrm{~km}$ entre le lieu de produc- tion et l'usine. Or, ils achètent le caoutchouc à un prix 22 à $50 \%$ inférieur au prix proposé par la CDC.

Enfin, deux autres facteurs se combinent aux conditions de commercialisation pour expliquer la baisse de production : le recours à de la main-d'œuvre rémunérée pour la saignée et l'évolution du prix du caoutchouc naturel.

Les conditions de commercialisation décrites ont un impact d'autant plus important que les paysans doivent disposer de trésoreries pour payer les saigneurs et garantir ainsi l'exploitation des hévéas. Cette situation est identique à celle du Gabon (Tran Van Canh et al., 2002), mais différente de celle de l'Asie du SudEst. On pourrait d'ailleurs supposer que, lorsque la main-d'œuvre familiale est mobilisée, la saignée peut être poursuivie. Or, les résultats d'enquête montrent que ce n'est pas le cas: les paysans se disent découragés par la culture et non motivés pour la saignée.

Le prix du caoutchouc payé aux producteurs a sans doute contribué à cette situation. L'étude a commencé à un moment où le cours du caoutchouc sur le marché mondial et donc le prix payé aux planteurs camerounais étaient particulièrement bas depuis plusieurs années (figure 2). Or, près de la moitié des planteurs interrogés se disent sensibles aux variations de cours du caoutchouc. Un prix élevé incite le chef d'exploitation, ou son fils, à saigner et l'encourage à chercher la main-d'œuvre nécessaire pour exploiter la totalité de la plantation. Les variations de prix ont aussi une influence sur le travail du saigneur. L'autre moitié des planteurs interrogés affirme que le niveau de prix n'a eu qu'une incidence limitée sur leurs pratiques d'exploitation des hévéas comparée au délai de paiement : ils ne connaissent pas le prix à l'avance et beaucoup ne suivent pas réellement son évolution. De plus, pour $90 \%$ des planteurs, un prix bas n'entraîne pas d'arrêt complet de la saignée. Le niveau de prix apparaît plus comme un facteur aggravant des conditions de commercialisation défavorables que comme un déterminant majeur de la faible production.

\section{Conclusion}

Les résultats présentés montrent bien que les conditions de commercialisation du 


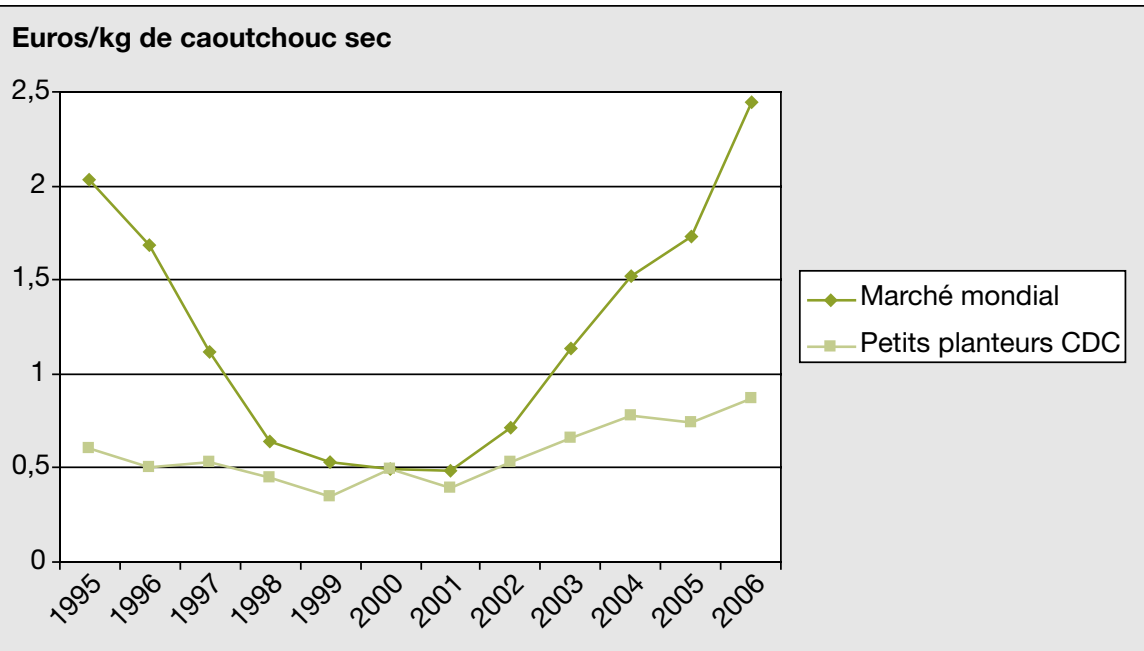

Figure 2. Évolution des cours du caoutchouc sur le marché mondial (SMR 20, Standard Malaysian Rubber) et du prix payé aux petits planteurs (d'après le Malaysian Rubber Board et CDC).

Figure 2. Natural rubber price trends on the world market (SMR 20) and paid to the smallholders (from the Malaysian Rubber Board and CDC).

CDC: Cameroon Development Corporation.

caoutchouc issu des plantations villageoises de la province Sud-Ouest du Cameroun sont un frein au développement de l'hévéaculture. La situation de monopole d'achat par une société agro-industrielle et la non-organisation de la filière créent des problèmes de trésorerie pour les planteurs et conduisent à une perte de revenu à court terme. Les difficultés ont été accentuées par le bas niveau de prix jusqu'en 2002. Les conséquences possibles de la forte remontée des cours du caoutchouc naturel depuis cette date restent à analyser.

L'accès au crédit, l'approvisionnement en matériel végétal amélioré de qualité, ou l'encadrement technique, sont d'autres conditions socio-économiques nécessaires au développement du secteur hévéicole villageois (Michels, 2001). Nous avons également vu que la création d'organisations de producteurs pourrait les aider notamment à mieux négocier et à valoriser leur production. Cependant, toutes ces conditions paraissent secondai- res par rapport à la commercialisation. En effet, garantir de bonnes conditions de commercialisation est une étape essentielle et préalable pour le développement des plantations villageoises d'hévéa. Toutes les améliorations qui pourraient être apportées pour promouvoir cette culture de rente n'auront qu'un impact limité, si les planteurs ne sont pas convaincus qu'ils pourront vendre leur production à un prix rémunérateur et être payés dans des délais acceptables.

\section{Références}

Buakaew S. Country report - Thaïlande. Proceedings of the ninth ANRPC seminar on the progress and development of rubber smallholders. Cochin (India), 9-11 November 2005.

Chambon B, Eschbach JM, Plaza C. Diagnostic du secteur hévéicole villageois au Cameroun. In : Penot E, Deheuvels O, eds. Modélisation économique des exploitations agricoles. Modélisation simulation et aide à la décision avec le logiciel Olympe. Paris : L'Harmattan, 2007.
Gobina MS, Chuba P, Chambon B. Study on the re-launching of rubber and oil palm in Cameroon. Report on rubber. Yaoundé (Cameroun): Irad; Cirad ; IITA; FAO, 2002.

Gouyon A, Supriono A. De la forêt à hévéas aux usines d'Akron : une production paysanne pour un marché industriel. In : Avenir des Zones tropicales humides. Montpellier: Cirad éditions, 1993.

Hashim O. Country report - Malaysia. Proceedings of the ninth ANRPC seminar on the progress and development of rubber smallholders. Cochin (India), 9-11 November 2005.

IRSG. Statistiques de production du caoutchouc naturel. Singapour: IRSG, 2007. www. rubberstudy.com

Kpolo M. Privatisation de la filière hévéa : états de lieux et enjeux pour un développement durable du secteur villageois. Proceedings de la conférence internationale sur "I'avenir des cultures pérennes. Investissement et durabilité en zones tropicales humides". Yamoussoukro (Côte-d'Ivoire), 5-9 novembre 2001.

Michels T. Petites et moyennes exploitations hévéicoles au Cameroun: systèmes de production, itinéraires techniques et stratégies. Diagnostic technico-économique, diagnostic agronomique et typologie. Ekona (Cameroun) : Irad ; Cirad ; Coopération française, 2001.

Plaza C. Situation de I'hévéaculture villageoise dans une filière en évolution : cas de la région d'Ekona, Province du Sud Ouest, Cameroun. Mémoire de fin d'études, Centre national d'études agronomiques des régions chaudes (Cnearc), Montpellier, 2003.

Place E. Vers le pré-usinage du caoutchouc des planteurs villageois au Cameroun : installation d'une unité pilote et diagnostic technicoéconomique. Montpellier: Cirad; ministère des Affaires étrangères, 2003.

Sardjono M. Country report - Indonesia. Proceedings of the ninth ANRPC seminar on the progress and development of rubber smallholders, Cochin (India), 9-11 November 2005.

Tran Van Canh, Eschbach JM, Penot E, Palu S. Caractérisation des exploitations hévéicoles au Gabon dans les provinces hévéicoles de Kango et Woleu-Ntem : analyse des résultats des enquêtes agronomiques, socioéconomiques et technologiques menées d'avril à juillet 2002. Rapport final. Montpellier: Cirad-CP, 2002.

Uhlan Haris, Teguh Wahyudi. Increasing farm gate price of smallholders' raw rubber material through appropriate marketing institution. Proceedings of IRRDB workshop on Transfer of Technology, Kuala Lumpur (Malaysia), 4-6 April 2005. 